\title{
Optical phase-conjugate correction for propagation distortion in nonreciprocal media
}

\author{
Sze-Keung Kwong \\ Ortel Corporation, 2015 West Chestnut Street, Alhambra, California 91803 \\ Ram Yahalom, Kazuo Kyuma, ${ }^{*}$ and Amnon Yariv \\ California Institute of Technology, Pasadena, California 91125
}

Received October 27, 1986; accepted February 5, 1987

\begin{abstract}
We demonstrate experimentally that the effects of nonreciprocal elements or media that would otherwise spoil phase conjugation can be neutralized by using a tandem combination of a modal-and polarization-scrambling fiber and a phase-conjugate mirror. A theoretical model to explain the experimental results is presented.
\end{abstract}

The basic property of time reversal and distortion correction by phase conjugation breaks down if the propagation path includes nonreciprocal media such as magnetic (or gyrotropic) components. This follows mathematically from the fact that the presence of imaginary elements in the expressions for the magnetic susceptibility tensor spoils the invariance of Maxwell equations under complex conjugation for the reflected wave. To illustrate the effect, consider the case shown in Fig. 1(a), where a plane wave initially with complex transverse components $\left(E_{x 1}, E_{y 1}\right)$ propagates through an element $A$, is phase conjugated, and returns to the initial plane after passing $A$ in reverse. If the element $A$ is a Faraday rotator with a Faraday angle $\theta$, the round trip is described by

$$
\begin{aligned}
& \left|\begin{array}{l}
E_{x} \\
E_{y}
\end{array}\right|_{4}=\left[\begin{array}{lr}
\cos \theta & -\sin \theta \\
\sin \theta & \cos \theta
\end{array}\right] \\
& \times \phi^{*}\left\{\left[\begin{array}{ll}
\cos \theta & \sin \theta \\
-\sin \theta & \cos \theta
\end{array}\right]\left[\begin{array}{l}
E_{x} \\
E_{y}
\end{array}\right]_{I}\right\} \\
& =\left[\begin{array}{rr}
\cos 2 \theta & -\sin 2 \theta \\
\sin 2 \theta & \cos 2 \theta
\end{array}\right]\left[\begin{array}{r}
-E_{x}{ }^{*} \\
E_{y}{ }^{*}
\end{array}\right]_{1},
\end{aligned}
$$

where we adopt the same mathematical notation presented in Ref. 1. $\phi^{*}(\quad)$ is an operator that represents the action of phase conjugation. The vector $\mathbf{E}_{4}$ is thus not the complex conjugate of $\mathbf{E}_{1}$.

If, on the other hand, the element $A$ is dielectric (reciprocal), say, a retardation plate tilted at $45^{\circ}$ with a retardation $\alpha$, then the round trip is described by

$$
\left|\begin{array}{l}
E_{x} \\
E_{y}
\end{array}\right|_{4}=\left[\begin{array}{rr}
\cos \frac{\alpha}{2} & -i \sin \frac{\alpha}{2} \\
-i \sin \frac{\alpha}{2} & \cos \frac{\alpha}{2}
\end{array}\right]
$$

$$
\begin{aligned}
& \times \phi^{*}\left\{\left[\begin{array}{rr}
\cos \frac{\alpha}{2} & i \sin \frac{\alpha}{2} \\
i \sin \frac{\alpha}{2} & \cos \frac{\alpha}{2}
\end{array}\right]\left[\begin{array}{l}
E_{x} \\
E_{y}
\end{array}\right]_{1}\right\} \\
= & {\left[\begin{array}{ll}
1 & 0 \\
0 & 1
\end{array}\right]\left[\begin{array}{r}
-E_{x}^{*} \\
E_{y}^{*}
\end{array}\right]_{1}, }
\end{aligned}
$$

and the effect of the (reciprocal) retardation plate is, as expected, canceled after a round trip.

In this Letter we describe a method to undo the nonreciprocal effect on polarization by employing a tandem combination of a polarization- and modalscrambling multimode fiber and a photorefractive phase-conjugate mirror [see Fig. 1(b)]. The experimental results are presented first, followed by a theoretical explanation.

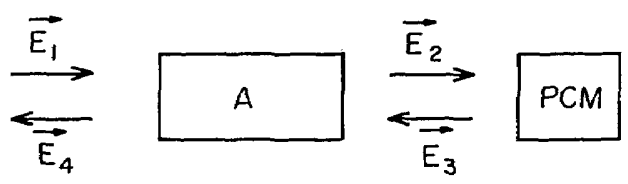

(a)

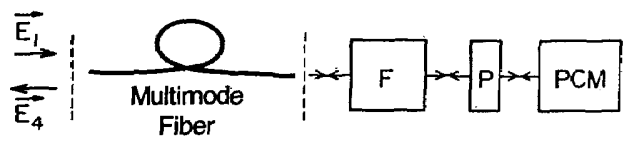

(b)

Fig. 1. (a) Schematic diagram of a wave that propagates through an element $\mathrm{A}$, is phase conjugated, and returns to the initial plane. (b) A method to undo the nonreciprocal effect. PCM, phase-conjugate mirror; P, polarizer; F, Faraday rotator. 

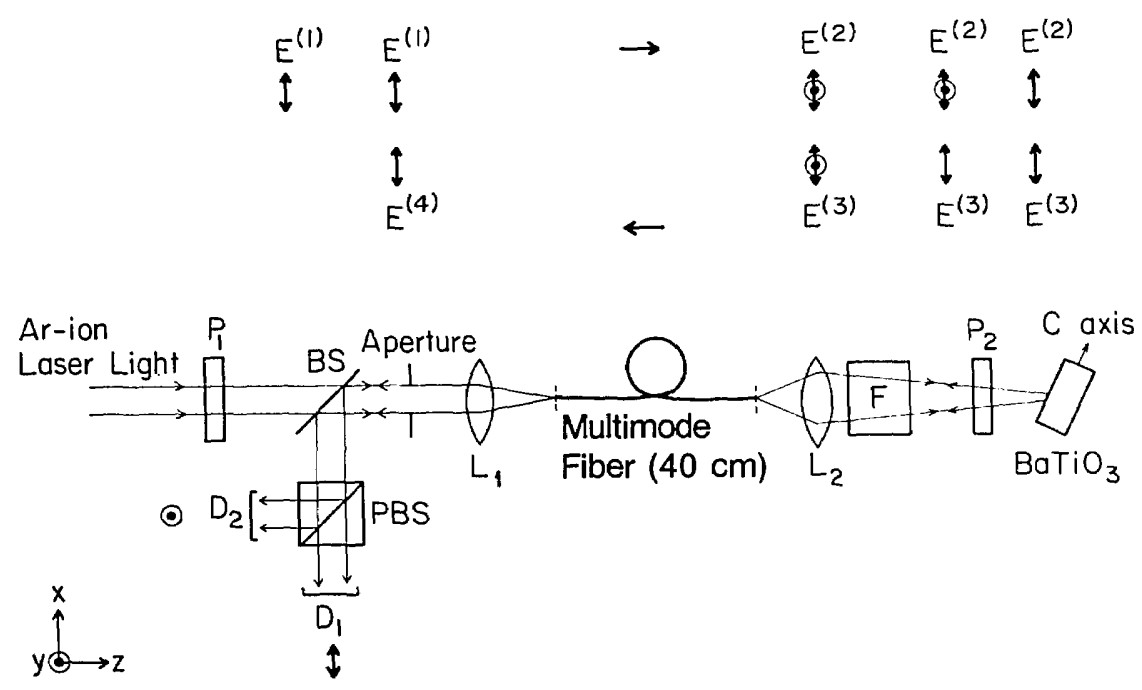

Fig. 2. The experimental arrangement used to demonstrate polarization recovery with a nonreciprocal medium. $\mathrm{P}_{1}, \mathrm{P}_{2}$, are $x$ polarizers; $B S$, beam splitter; PBS, polarizing beam splitter; $\mathrm{L}_{1}, \mathrm{~L}_{2}$, lenses; $\mathrm{D}_{1}, \mathrm{D}_{2}$, photodetectors; $\mathrm{F}$, variable Faraday rotator.

The experimental arrangement is shown in Fig. 2. The output light from the multilongitudinal-mode argon-ion laser $(\lambda=514.5 \mathrm{~nm}$, beam diameter $2 \mathrm{~mm}$, linear polarized along the $x$ axis) was coupled into a graded-index multimode fiber (100- $\mu \mathrm{m}$ core diameter, $40 \mathrm{~cm}$ in length). The output of the fiber was focused onto a $\mathrm{BaTiO}_{3}$ crystal, which was aligned to form a self-pumped phase-conjugate mirror. ${ }^{2}$ A variable Faraday rotator was placed behind lens $\mathrm{L}_{2}$. A polarizer was interposed between the Faraday rotator and the crystal in order to obtain linear-polarized light at the input to the crystal. The reflected phase-conjugate light was also linearly polarized (along the $x$ axis), passed through the Faraday rotator, and coupled into the fiber automatically. Part of the phase-conjugate light was picked off by a polarization-independent beam splitter BS. A 2-mm-diameter aperture was used to eliminate backreflections from the optical components. The degree of the polarization recovery was measured and is characterized by the quantity $p=$ $\left(P_{1}-P_{2}\right) /\left(P_{1}+P_{2}\right)$, where $P_{1}$ and $P_{2}$ are, respectively, the optical power of the two orthogonal polarization components of $\mathbf{E}^{(4)}$. When the polarization state is recovered perfectly, the quantity $p$ is unity.

The experimental data are plotted in Fig. 3. We observed that

(1) Both the spatial information and the original state of polarization are recovered. The quantity $p$ is nearly unity from $\theta=20^{\circ}$ to $\theta=48^{\circ}$. (The range of Faraday rotation angles is limited by the variable Faraday rotator.)

(2) The intensity of the phase-conjugate beam decreases approximately as $\cos ^{2}(2 \theta)$. The phase-conjugate reflectivity, which was monitored by a beam splitter, was constant throughout the experiment.

In what follows, we extend the theoretical model proposed in Ref. 3 to explain the above observations. We adopt the symbols and conventions introduced in Refs. 1, 3, and 4. By using a circular polarization representation, the input field to the fiber can be expressed as

$$
\begin{aligned}
\mathbf{E}^{(1)} & =\sum_{n=1}^{N}\left(a_{R n}^{(1)} E_{R n}+a_{L n}^{(1)} E_{L n}\right) \\
& =\left[\begin{array}{c}
a_{R 1}^{(1)} \\
\vdots \\
a_{R N}^{(1)} \\
a_{L 1}^{(1)} \\
\vdots \\
a_{L N}^{(1)}
\end{array}\right],
\end{aligned}
$$

where $E_{R n}$ and $E_{L n}$ are the $n$th right-hand and lefthand circular fiber modes, respectively. The phaseconjugate beam $\mathbf{E}^{(4)}$ can be written as

$$
\mathbf{E}^{(4)}=M^{\prime} F^{\prime} P^{\prime} \phi^{*}\left[P F M E^{(1)}\right]
$$

where

$M=\left[\begin{array}{ll}M_{R R} & M_{R L} \\ M_{L R} & M_{L L}\end{array}\right], \quad M^{\prime}=\left[\begin{array}{ll}M_{R R}^{\prime} & M_{R L}^{\prime} \\ M_{L R^{\prime}} & M_{L L}^{\prime}\end{array}\right]$

are the $2 N$-rank polarization- and mode-scrambling matrices for traveling forward or backward along the fiber;

$$
F=\left(\begin{array}{lr}
e^{i \theta} I & 0 \\
0 & e^{-i \theta} I
\end{array}\right), \quad F^{\prime}=\left(\begin{array}{lr}
e^{-i \theta} I & 0 \\
0 & e^{i \theta} I
\end{array}\right)
$$

are $2 N$-rank Faraday rotation matrices for traveling along and opposite the magnetic field, respectively. In Eqs. (6) we assume that all fiber modes suffer the same amount of Faraday rotation, $\theta$.

$$
P=\frac{1}{2}\left[\begin{array}{ll}
I & I \\
I & I
\end{array}\right]=P^{\prime}
$$

is the matrix for the linear polarizer. 


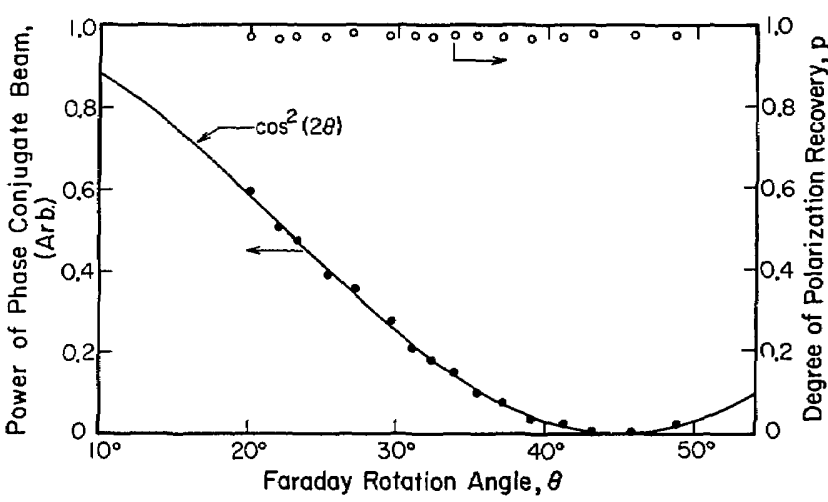

Fig. 3. Experimental results: open circles, degree of polarization; closed circles, power of phase-conjugate beam. The solid curve is from the theoretical model.

Substituting Eqs. (5)-(7) into Eq. (4), we obtain
From Eqs. (9) we notice that the spatial information and the polarization are recovered. The intensity of the phase-conjugate beam varies as $\cos ^{2}(2 \theta)$, in agreement with the experimental data of Fig. 3. To simulate distributed Faraday rotation in a fiber better, the theory was generalized to a sequence of $N$ Faraday rotators separated by sections of fiber. The result, which was also proved experimentally, is

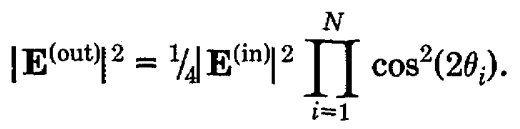

More detailed analysis of the effect of a magnetic field on optical fiber in the presence of a phase-conjugate mirror and its potential applications will be reported in a forthcoming publication.

In conclusion, we have demonstrated both experimentally and theoretically a method based on a tandem combination of a modal- and polarization-scram-

$$
\begin{aligned}
\mathbf{E}^{(4)}= & \frac{1}{2}\left\{\begin{array}{l}
M_{R R} M_{R R}{ }^{*} e^{-i 2 \theta}+M_{R R}{ }^{\prime} M_{L R}{ }^{*}+M_{R L} M_{R R}{ }^{*}+M_{R L}{ }^{\prime} M_{L R} e^{2 i \theta} \\
M_{L R^{\prime}} M_{R R}{ }^{*} e^{-21 \theta}+M_{L R^{\prime}} M_{L R}{ }^{*}+M_{L L} M_{R R}{ }^{*}+M_{L L} M_{L R} e^{2 i \theta}
\end{array}\right. \\
& \left.\times \begin{array}{l}
M_{R R^{\prime}} M_{R L}{ }^{*} e^{-i 2 \theta}+M_{R R^{\prime}} M_{L L}{ }^{*}+M_{R L} M_{R L}{ }^{*}+M_{R L}{ }^{\prime} M_{L L}{ }^{*} e^{2 i \theta} \\
M_{L R} M_{R L}{ }^{*} e^{-21 \theta}+M_{L R} M_{L L}{ }^{*}+M_{L L} M_{R L}{ }^{*}+M_{L L} M_{L L} e^{2 i \theta}
\end{array}\right\}\left(\mathbf{E}^{(1)}\right)^{*} .
\end{aligned}
$$

From the fact that, in the absence of Faraday rotator, a complete polarization recovery is achieved, ${ }^{4}$ we know that

$$
M_{R R^{\prime}} M_{R R}{ }^{*} \sim M_{R L}{ }^{\prime} M_{L R}{ }^{*} \sim M_{L R}{ }^{\prime} M_{R L}{ }^{*} \sim M_{L L}{ }^{\prime} M_{L L}{ }^{*} \sim 1 / 2 I
$$

and that

$$
\begin{aligned}
M_{R R}{ }^{\prime} M_{L R}{ }^{\prime} \sim & M_{R L}{ }^{\prime} M_{R R}{ }^{*} \sim M_{L R}{ }^{\prime} M_{R R}{ }^{*} \sim M_{L R}{ }^{\prime} M_{L R}{ }^{*} \\
& \sim M_{L L}{ }^{\prime} M_{R R}{ }^{*} \sim M_{L L} M_{L R}{ }^{*} \sim M_{R R^{\prime}} M_{R L}{ }^{*} \\
& \sim M_{R R}{ }^{\prime} M_{L L}{ }^{*} \sim M_{R L}{ }^{\prime} M_{R L}{ }^{*} \sim M_{R L}{ }^{\prime} M_{L L}{ }^{*} \\
& \sim M_{L R}{ }^{\prime} M_{L L}{ }^{*} \sim M_{L L}{ }^{\prime} M_{R L}{ }^{*}=0 .
\end{aligned}
$$

These relations were shown ${ }^{3}$ to be true in the case of strong mode coupling, i.e., when the energy is distributed equally among all modes, and $M_{i j}$ can be written as $(1 / \sqrt{N}) e^{i \phi_{i j}}$. We therefore get

$$
\begin{aligned}
\mathbf{E}^{(4)} & =1 / 4\left(e^{2 i \theta}+e^{-2 i \theta}\right) I\left(\mathbf{E}^{(1)}\right)^{*}, \\
\mathbf{E}^{(4)} & =1 / 2 \cos 2 \theta\left(\mathbf{E}^{(1)}\right)^{*} .
\end{aligned}
$$

bling fiber and a phase-conjugate mirror to correct the effect of nonreciprocal media.

This research is sponsored by the U.S. Army Research Office (R. Guenther) and the U.S. Air Force Office of Scientific Research (H. Schlossberg). The authors would like to acknowledge helpful discussions with Y. Tomita and M. Cronin-Golomb.

* Permanent address, Central Research Laboratory, Mitsubishi Electric Corporation, 8-1-1 Tsukaguchi-Honmachi, Amagasaki, Hyogo 661, Japan.

\section{Reference}

1. A. Yariv, "Operator algebra for propagation problems involving phase conjugation," submitted to Opt. Lett.

2. J. Feinberg, Opt. Lett. 7, 486 (1982).

3. A. Yariv, Y. Tomita, and K. Kyuma, Opt. Lett. 11, 809 (1986).

4. K. Kyuma, A. Yariv, and S-K. Kwong, Appl. Phys. Lett. 49, 617 (1986). 